\title{
Tropical cyclones in the Bay of Bengal and deterministic methods for prediction of their trajectories
}

\author{
U C MOHANTY
}

Centre for Atmospheric Sciences, Indian Institute of Technology, Hauz Khas, New Delhi 110016, India

Present address: National Centre for Medium Range Weather Forecasting, IMD Complex, Lodi Road, New Delhi 110003, India

\begin{abstract}
Based on information about tropical storms and depressions in the Bay of Bengal over a 100-year period (1877-1976), certain climatic characteristics of tropical cyclones are examined. A brief description of climatic parameters, notably the region of their development, direction and nature of movement and percentage of disturbances intensified into severe tropical storms in different seasons of the year, which are explicitly or implicitly related to the formulation of objective methods for forecasting storm tracks, is presented in this work.

A brief review of objective methods (numerical and statistical) used for forecasting the tracks of cyclonic storms and the rationale for development of such methods are described. Two numerical methods based on integral and steering flow concepts and four different physical-statistical methods, which take into consideration the influence of both external and internal forces responsible for the movement of tropical cyclones, are developed for forecasting the trajectories of post monsoon cyclonic storms in the Bay of Bengal.

Performance of these six objective methods are illustrated through the study of a homogeneous sample of cyclonic storms (14 cases) in the Bay of Bengal during the post monsoon seasons of 1975 and 1976. These results are compared with those obtained from some of the existing methods for forecasting the movement of tropical cyclones in the Bay of Bengal. Finally, the limitations and prospects of objective methods in predicting the tracks of cyclonic storms in the Bay are discussed.
\end{abstract}

Keywords. Bay of Bengal; tropical cyclones; storm tracks; deterministic methods; numerical weather prediction.

\section{Introduction}

The Bay of Bengal is a potentially energetic region for the development of cyclonic storms, accounting for about $7 \%$ of the global annual total number of tropical storms (Gray 1968). Furthermore, the Bay storms are exceptionally devastating, especially 
when they cross over into the coastal states of India and Bangladesh (De Angelis 1976). However, inadequate present-day knowledge about their physical mechanisms of development, evolution and movement and deficient observational data close to the radius of influence of cyclonic storms limit the degree of physical and mathematical sophistication and thus make it difficult to develop more accurate objective methods for forecasting their movements.

Occurrence of cyclonic storms in the Bay of Bengal continues to be of great concern to India and Bangladesh and prediction of their tracks is one of the most challenging problems in meteorology.

The purpose of the present study is to investigate the problem of predicting the tracks of tropical storms in the Bay of Bengal using objective (numerical and statistical) techniques. First, a brief account of certain climatic characteristics of tropical cyclones in the Bay of Bengal are presented which provide some guidelines for the formulation of objective methods. Compared to other seasons, the post-monsoon season storms in the Bay of Bengal are more frequent and devastating in nature and a fair number of them have recurving tendencies, which make the forecasting of their evolving trajectories extremely difficult. Two numerical and four statistical methods have been developed for forecasting the track of post-monsoon season tropical storms. Performances of these six objective methods are illustrated, based on a homogeneous sample of cyclonic storms that occurred in the Bay of Bengal during the years 1975 and 1976.

\section{Climatology of tropical cyclones}

Climatology of the development and movement of cyclonic storms is quite helpful in the process of formulating objective methods for forecasting their tracks. The frequency, location of the initially observed storms and nature of their movements are some of the important factors which are considered in this work.

For this study, information about the tracks of the storms and depressions in the Bay of Bengal for 100 years (1877-1976) were collected from India Meteorological Department publications. Data for the first 94 years were obtained from the India Met. Dept. (1979) atlas and for the remaining 6 years (1971-1976) from other publications.

Cyclonic disturbances are classified into three categories as Tropical Depressions (TD), Tropical Storms (TS) and Severe Tropical Storms (STS) depending on their maximum sustained winds by various systems as defined by the India Meteorological Department (India Met. Dept. 1979). In this study TD stands for all the cyclonic disturbances which attained the stage of a tropical depression (TD) and thus includes TS and STS. TS includes all cyclonic disturbances in which the maximum sustained winds were $34 \mathrm{kt}$ (i.e. $63 \mathrm{~km} / \mathrm{h}$ ) or higher. Further, a year was divided into four seasons, each of 3 months duration. The period January to March is a passive period for cyclonic disturbances. April to June is considered the pre-monsoon period, July to September, the monsoon, and October to December, the post-monsoon period, though the actual monsoon season lasts from June to September.

Figure 1 shows the annual percentage distribution of TD, TS and STS for a period of 100 years. More than $60 \%$ of all cyclonic disturbances occur between July and October with a maximum in September, whereas only about $2 \%$ occur between January and March. The cyclonic storms show three maxima, with the primary one 


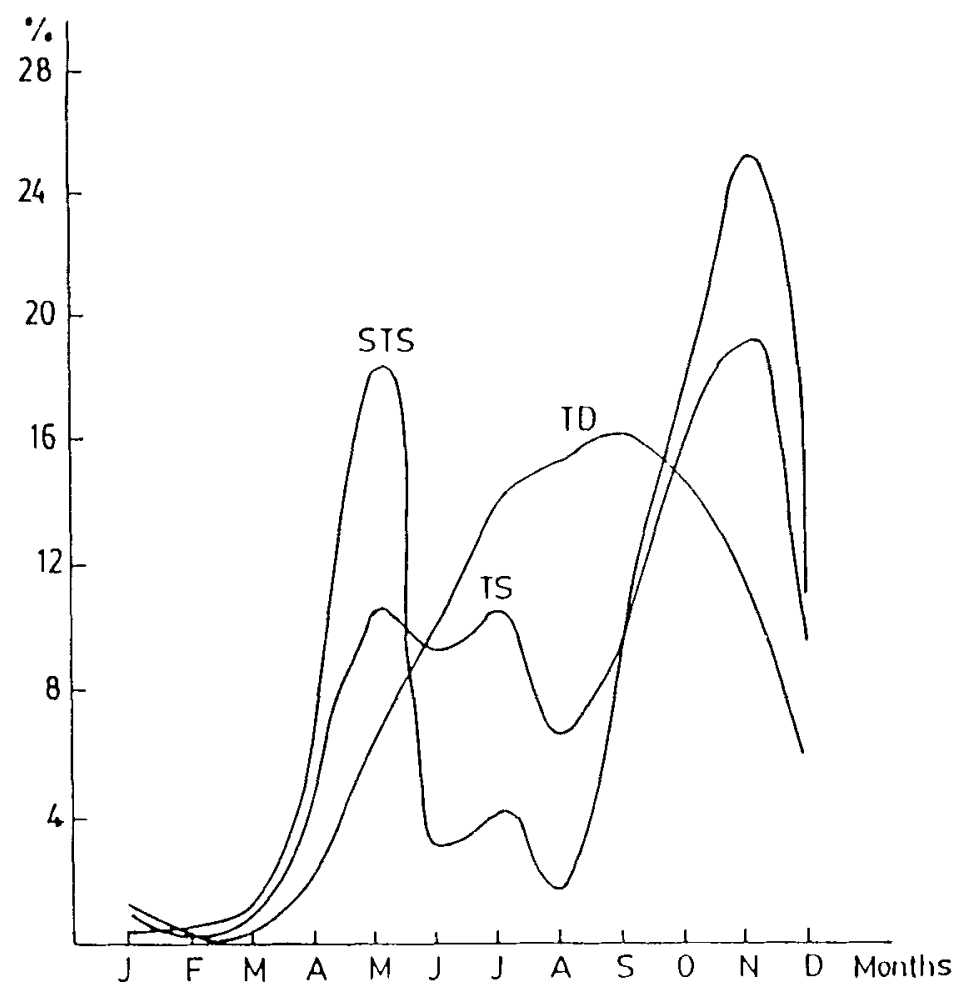

Figure 1. Percentage distribution of the occurrence of tropical disturbances (TD), tropical storms (TS) and severe tropical storms (STS) relative to calendar year.

occurring in the month of November $\left(19 \cdot 5^{\circ}{ }_{0}\right)$. Severe cyclonic storms exhibit two sharp maxima with the primary one (more than $25 \%$ ) occurring in November and another (about $19 \%$ ) in May.

Some of the interesting conclusions based on these results are as follows :

i) January to March is a passive period for cyclonic disturbances and thus will not figure in further discussions.

ii) The most active period of tropical depressions (TD) is the summer monsoon period (July-September). However, this period is quite passive for the occurrence of TS and STS, as only 2 to $7_{\%}^{\circ}$ of the depressions intensify into TS and STS.

iii) The maximum number of cyclonic storms (about $46 \%$ ) occur in the post-monsoon period (October-December). This is also the period of generation of the maximum number of severe tropical storms (more than $54 \%$ ) in the Bay of Bengal.

iv) In general, there are two severe cyclonic storm seasons in the Bay of Bengal. The primary one is the post-monsoon period $(54.5 \%)$ and the second, the pre-monsoon season with $27.6 \%$ STS.

Figure 2 illustrates seasonal variations of the location (latitude- $\phi$ and longitude- $\lambda$ ) at which initial cyclonic disturbances, which later became TS and STS, were detected. It is seen that the location of initially observed cyclonic disturbances is further northward $\left(18^{\circ}-20^{\circ} \mathrm{N}\right)$ during the monsoon season compared to the pre- and post-monsoon periods $\left(8^{\circ}-12^{\circ} \mathrm{N}\right)$. Such a latitudinal migration of the location of the occurrence of cyclonic storms agrees well with the latitudinal migration of the equatorial trough 


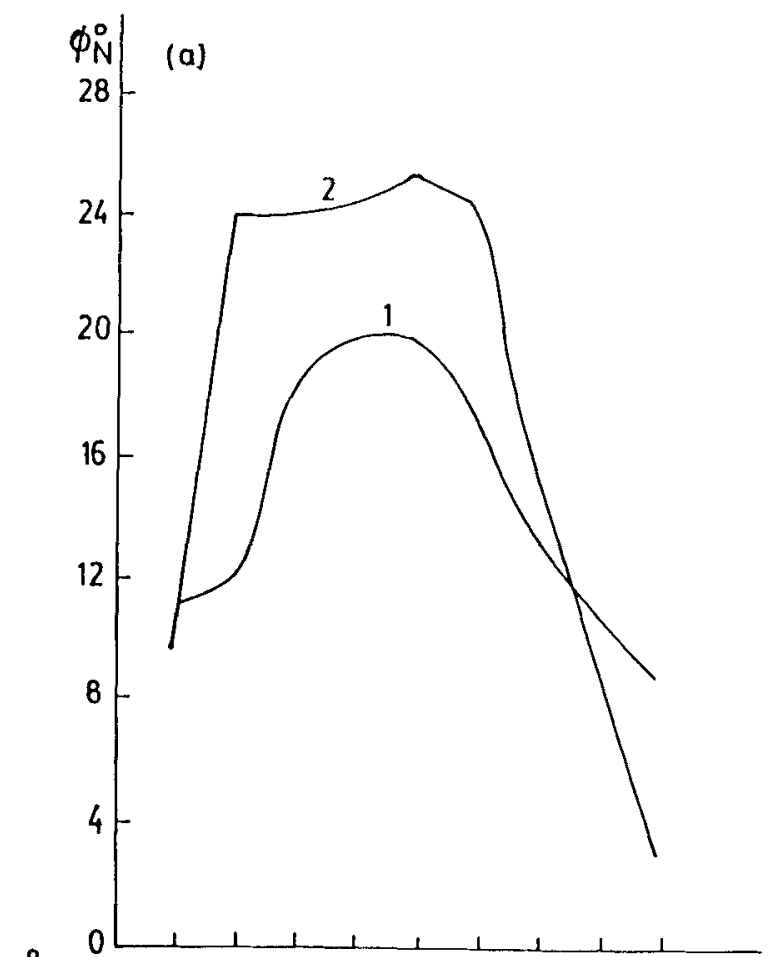

$\lambda{ }^{\circ} E$

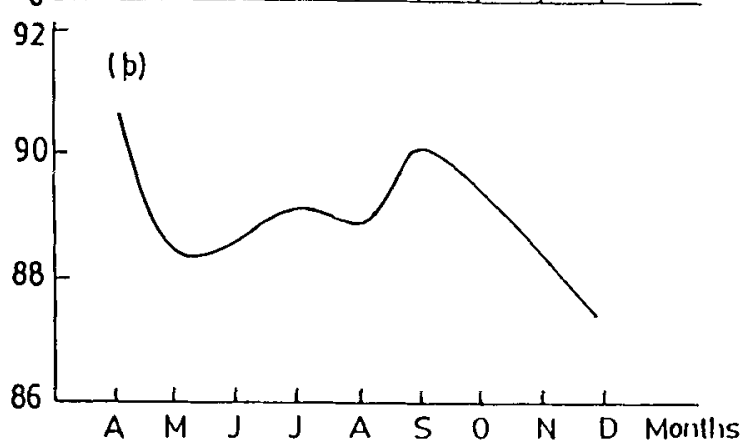

Figure 2. Seasonal $(\mathbf{a}, 1)$ latitude and (b) longitude variations of initially located cyclonic disturbances which later became tropical storms. (a, 2 corresponds to the seasonal latitude variation of the equatorial trough from Gray 1968.)

(Gray 1968) and the inter-tropical convergence zone (Minina \& Niguen 1969). Seasonal longitudinal variations of the location of cyclonic disturbances which later became TS and STS is not large $\left(87-90^{\circ} \mathrm{E}\right.$ ). From figures 1 and 2 (curve-1), we arrive at the interesting conclusion that if a cyclonic disturbance originates in the Central Bay of Bengal $\left(9-10^{\circ} \mathrm{N}\right.$ and $\left.88-90^{\circ} \mathrm{E}\right)$, then the probability of its intensification into TS and STS becomes very high (40 to $80 \%$ ). On the other hand, the probability of intensifications of a cyclonic disturbance into TS and STS decreases considerably ( 2 to $15 \%$ ) if it initially forms in the northern part of the Bay $\left(18-20^{\circ} \mathrm{N}\right.$ and $88-89^{\circ} \mathrm{E}$ ).

For further detailed study, computation of the mean $(\mu)$, standard deviation $(\sigma)$, coefficient of variation $(c v)$, skewness $(S)$, kurtosis $(K)$ and also standard error of skewness $\left(\sigma_{s}\right)$ and kurtosis $\left(\sigma_{k}\right)$ of the initial location (latitude and longitude) of cyclonic disturbances which intensified into TS and STS, were carried out by Mohanty $\&$ Dube (1981) with a 100-year data set. The results are given in table 1 . 

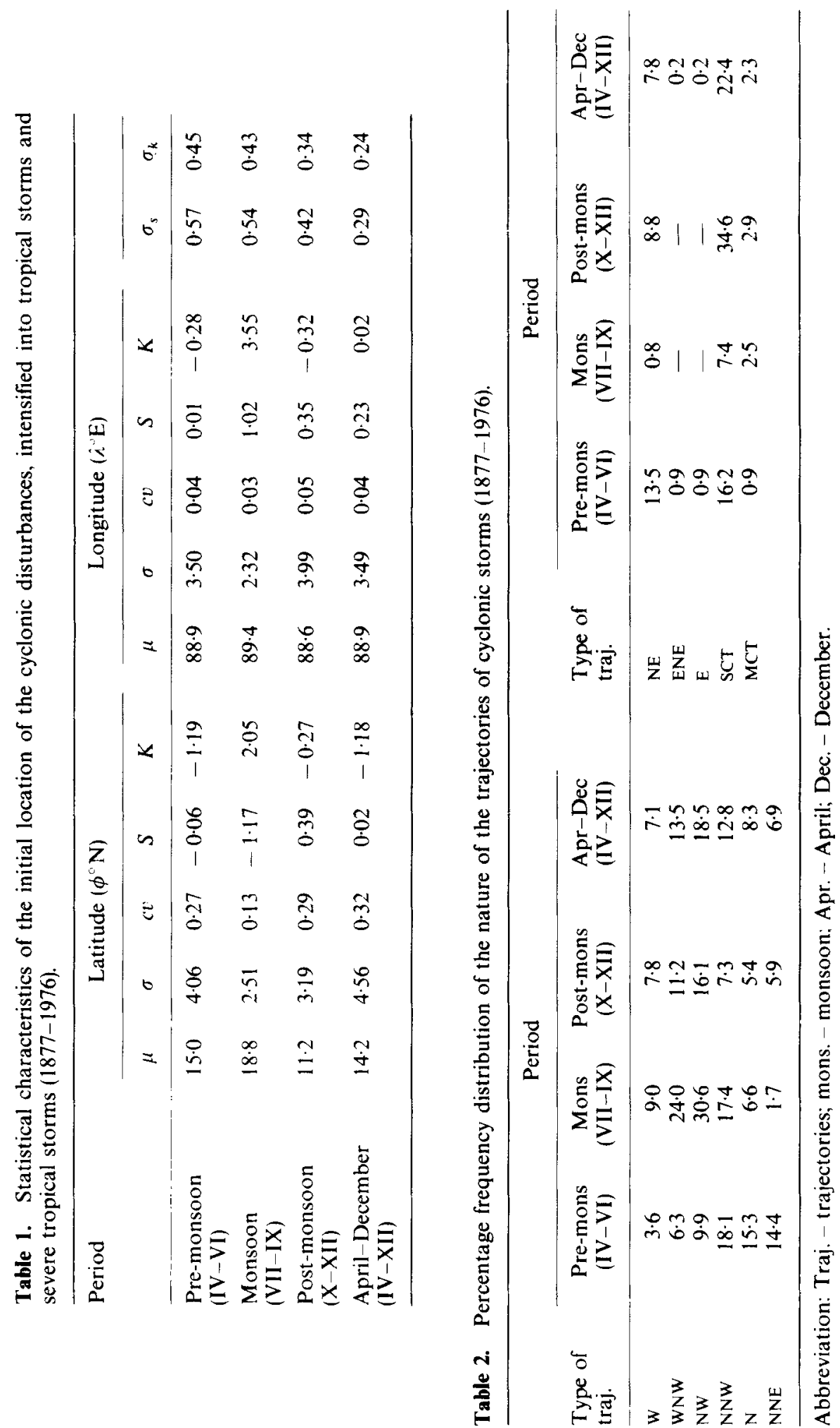
From the results in table 1, the following conclusions are drawn:

(i) During the monsoon period, latitudes of initially observed cyclonic disturbances, which later became TS and STS, are more homogeneous compared to the pre- and post-monsoon periods, as both the standard deviation $(\sigma)$ and the coefficient of variation $(c v)$ of $\phi$ during the monsoon period are the least $\left(2.51^{\circ}\right.$ and $0.13^{\circ}$ respectively).

(ii) The coefficient of variation of longitude is very small (less than $0.05^{\circ}$ ) during all the three seasons. This implies that the longitude of the origin of cyclonic disturbances is more homogeneous compared to the latitude.

(iii) From skewness and kurtosis of $\phi$ and $\lambda$ and their standard errors, it is clear that during the post-monsoon period, the skewness and kurtosis of both $\phi$ and $\lambda$ do not exceed $\sigma_{s}$ and $\sigma_{k}$ respectively. Initial locations of the post-monsoon cyclonic disturbances which later intensify into TS and STS are thus symmetrical about the mean position $\left(11.2^{\circ} \mathrm{N}\right.$ and $\left.88.6^{\circ} \mathrm{E}\right)$ and follow the normal laws of distribution.

According to the nature of their trajectories, TS and STS may be broadly divided into two categories, namely, the storms with simple trajectories (if movement of TS and STS is almost in one direction) and those with parabolic trajectories (i.e. movement of TS and STS in a recurved path). About $75 \%$ of the cyclonic storms in the months of April to December belong to the first category and the remaining $25 \%$ storms exhibit tracks with one or more points of recurvature.

For a detailed account of the nature of movement of cyclonic storms, those belonging to the first category were further divided into 9 classes according to their direction of movement on 16 points of the compass as they do not move into the southern sector (i.e. West-South West to East-South East). Cyclonic storms of the second group were divided into two classes: storm tracks with a single point of recurvature (SCT) and those with two or more points of recurvature (MCT).

Table 2 gives the seasonal percentage frequency of the nature of trajectories of cyclonic storms which are divided into 11 classes as stated above. The results in table 2 lead to the following conclusions:

(i) During April to December, the maximum number of cyclonic storms $(22.4 \%)$ exhibit tracks with a point of recurvature and a smaller number $(18.5 \%)$ move in the North-West $(\mathrm{NW})$ direction. Over a 100 -year period, the number of cyclonic storms which moved in the East-North East (ENE) and East (E) directions were only two (about $0.4 \%$ ) whereas those that moved in the West-North-West (WNW), North-West (NW) and North-North-West (NNW) directions constituted more than $45 \%$.

(ii) The nature of the tracks of TS and STS vary from season to season. In the pre-monsoon period, more than $60 \%$ of the storms move in the NWN, N, NNE and NE directions, with a maximum number of storms $(18.1 \%)$ in the NNW direction. During the monsoon period, more than $80 \%$ of the cyclonic storms move in $\mathrm{W}, \mathrm{WNW}, \mathrm{NW}$ and $\mathrm{NNW}$ directions, the majority $(30.6 \%)$ in the $\mathrm{NW}$ direction.

(iii) Maximum number (34.5\%) of cyclonic storms occur in the post-monsoon period and over $72 \%$ of these are observed to recurve their tracks. Only $18 \%$ and $10 \%$ of such storms occur in the pre-monsoon and monsoon periods respectively.

These results on the climatology of tropical cyclones in the Bay of Bengal show 
that the maximum number of tropical storms (more than $46 \%$ ) and severe tropical storms (more than $54 \%$ ) occur in the post-monsoon period. Also the maximum number of (more than $72^{\circ} \%$ ) recurved tracks of cyclonic storms appear during this period of the year.

The task of predicting the recurvature of a storm's track constitutes a difficult problem and most of the forecasting models fail at this point. Therefore an attempt has been made in this work to develop some objective techniques to predict the movement of cyclonic storms in the Bay of Bengal during the post-monsoon period.

\section{Objective methods of forecasting the movement of cyclonic storms}

Objective methods have made significant contributions to the theory and practice of weather forecasting on all time scales. In general, objective methods are divided into two classes : numerical (i.e. dynamical) methods and statistical methods. Both these methods are widely used to provide routine objective guidance on prediction of various atmospheric phenomena. Though dynamical methods are based on the laws of physics and mathematical theories and are found to be quite successful in forecasting large-scale atmospheric processes in the mid-latitudes, their performance is not very satisfactory in predicting the movement of tropical cyclones. This is mainly due to inadequate information available about tropical cyclones as well as lack of adequate understanding of the physical mechanism of their development, evolution and movement. Performance of present day statistical methods in predicting the movement of tropical storms is therefore better or at least comparable with those of numerical methods. Further, statistical methods are simple and economical. As such, a survey of the worldwide objective prediction models by Hope \& Neumann (1977) cites 31 operational models, most of which are statistical models. However, future progress in prediction models to provide objective guidance for routine forecasts of the tracks of cyclones will depend heavily on dynamical methods. Clearly the most reliable forecasts in the future will be produced by approaches or procedures that make judicious use of both numerical and statistical methods.

A brief account of a number of dynamical and statistical methods, developed to predict the tracks of post-monsoon cyclonic storms in the Bay of Bengal, is given below.

\section{$3.1 \quad$ Numerical methods}

Based on the concept of the circulation patterns in and around a tropical cyclone, the numerical (i.e. dynamical) methods for forecasting the movement of storm tracks are generally divided into two broad groups, the steering flow method (external forces) and the integral method (internal as well as external forces). In this work attempts have been made to present two dynamical models which belong to the above two groups.

Further, an attempt has also been made to present more complex numerical models based on multi-level primitive equations with appropriate physical processes incorporated in parameterization form. Such models are used for the prediction of synoptic scale atmospheric processes including intense atmospheric vortices (such as cyclones, depressions, western disturbances etc). However, such models require a knowledge of more comprehensive, dense and high quality meteorological fields as initial conditions for integration. 
3.1a Steering flow method: In this method, the cyclonic storm is represented by a constant circular vortex stream described in terms of the maximum sustained wind and the radius of the storm's eye of its influence. Also barotropic flow is assumed. Further, the vortex of the tropical cyclone is eliminated from the initial stream function analysis leaving only a steering wind field in the region of the cyclone. The cyclonic vortex motion is estimated by the steering current which is determined by a numerical model. This concept was used for forecasting the tracks of cyclonic storms by Kasahara (1959), Sitnikov (1968), and others.

A brief description of this method is given here and one may refer to Kivganov \& Mohanty $(1979 b)$ for details. In this method the total barotropic stream function $(\psi)$ is considered to be the sum of a steering stream function $(\bar{\psi})$ and a local cyclonic vortex stream function $\left(\psi^{*}\right)$, that is

$$
\psi=\bar{\psi}+\psi^{*}
$$

Further, $\psi^{*}$ is divided into two components as

$$
\psi^{*}=\psi_{1}^{*}+\psi_{2}^{*}
$$

where $\psi_{1}^{*}$ is the vortex stream function due to tangential wind $V_{\theta}$ and $\psi_{2}^{*}$ is the stream function due to translational motion $v_{0}$ of the storm. For a detailed description of the computation of $\psi_{1}^{*}$ and $\psi_{2}^{*}$, one may refer to Kivganov \& Mohanty (1979b).

The total barotropic stream function $(\psi)$ is computed from the horizontal components of the wind (Mohanty \& Madan 1983), and the steering stream function $(\bar{\psi})$ is estimated by (1).

The instantaneous speed of the movement of the centre of a cyclonic storm can be computed from the steering stream function $(\bar{\psi})$ by the following relations (Mohanty 1978).

$$
\begin{aligned}
& C_{x o}=-m \frac{\partial \bar{\psi}}{\partial y}-\frac{m}{\mu^{2}} \frac{\partial}{\partial y}\left(m^{2} \nabla^{2} \bar{\psi}+f\right) \\
& C_{y o}=m \frac{\partial \bar{\psi}}{\partial y}-\frac{m}{\mu^{2}} \frac{\partial}{\partial y}\left(m^{2} \nabla^{2} \bar{\psi}\right) \\
& V_{0}=\left(C_{x o}^{2}+C_{y o}^{2}\right)^{1 / 2}
\end{aligned}
$$

where $C_{x o}$ and $C_{y o}$ are the speed of movement of the centre of the storm along the $x$ and $y$-axes respectively, $m$ is the map factor, $f$ is the Coriolis parameter, and $\mu$ is a characteristic parameter of the horizontal dimension of the storm $(\mu R=3.83)$ and $\nabla^{2}$ is the Laplacian operator.

For forecasting the movement of the cyclonic storm using (3), the steering stream function is predicted by a modified non-divergent barotropic model,

$$
\left(m^{2} \nabla^{2}-\alpha^{2}\right)(\partial \bar{\psi} / \partial t)=m^{2} J\left(m^{2} \nabla^{2} \psi+f, \bar{\psi}\right)+9 m^{2} \nabla^{2}\left(m^{2} \nabla^{2} \bar{\psi}\right)
$$

where $\alpha^{2}$ is a constant $\left(\alpha^{2}=f^{2} / R \bar{T}\right), R$ the gas constant for air, $\bar{T}$ the mean tropospheric temperature, $\vartheta$ the kinematic coefficient of eddy viscosity and $J$ the Jacobian operator.

This method for forecasting the movement of the centre of the cyclone involves two steps. At each time step a predicted value of the steering stream function $(\bar{\psi})$ is obtained by solving the modified vorticity equation (4). In the next step the predicted 
value $\bar{\psi}$ is used to obtain the instantaneous speed and direction of the movement of the centre of the storm by relation (3).

3.1b Integral method: In this method, the cyclonic storm is considered an inseparable part of the large-scale flow and thus free interaction of the cyclonic storm with its outer environment is allowed. The predicted path of the tropical storm results from tracking its centre as a minimum geopotential height/stream function value at a constant pressure level. This method is widely used for forecasting the movement of tropical storms (Sanders et al 1975, Sikka 1975, Singh \& Saha 1976, and others). In general either barotropic vorticity equation or primitive equation forecasting model is used for the integral method.

In the present study we have used the primitive equation barotropic model for forecasting the movement of post-monsoon storms in the Bay of Bengal. The model equations, initialization procedure and numerical solution of this model are described in an earlier work by Mohanty (1982). This model is used to predict the geopotential height and horizontal wind fields. Based on the analyses of these forecasted fields the centre of the storm and thus its future trajectory are determined.

3.1c Complex atmospheric models: In recent years, with the advances in high speed computers, more complex atmospheric models have been developed for simulation/ prediction of large-scale atmospheric phenomena. They are broadly of two categories: (i) Limited area models (LAM) for a specified region with a capability to integrate over a shorter period of 1-2 days to obtain short-range prediction of the regional features in greater detail, and (ii) general circulation models (GCM) for the entire globe with capability to integrate for any length of time as they are not influenced by artificial lateral boundary conditions. GCM is generally used for extended range simulation/prediction over the entire globe.

Both LAM and GCM can be used to predict the movement of tropical cyclones and other atmospheric vortices (such as lows, depressions and deep depressions) based on the concept of integral methods (as discussed in $\$ 3 \cdot 1 \mathrm{~b}$ ).

For the sake of completeness a brief description of each of the LAM and GCM is presented. As an example of the performance of these models, an illustration of the prediction of storm tracks is also presented.

(i) Limited area numerical weather prediction model (Mohanty et al 1989) - In this study a primitive equation ( $\mathrm{PE}$ ) model governing the motion of the atmosphere, appropriate to a meso-scale quasi-hydrostatic baroclinic system is considered. The model with five prognostic and two diagnostic equations (viz. the zonal and meridional momentum, the thermodynamic, the moisture continuity, the surface pressure tendency, the hydrostatic and the mass continuity equations) for the seven basic meteorological variables forms a closed system and the model equations are represented in flux form, in spherical coordinates. The model uses sigma coordinates in the vertical and incorporates topography and physical parameterizations of the planetary boundary layer, air-sea exchange of heat and moisture and precipitation processes. Details of the model as well as numerical solution of the model equations and initialization procedure of mass and velocity fields in the model are presented in earlier work by Mohanty et al (1988, 1989).

As an illustration of the performance of the model, the actual track of a monsoon depression in the Bay of Bengal during intensive observation periods of the monsoon 


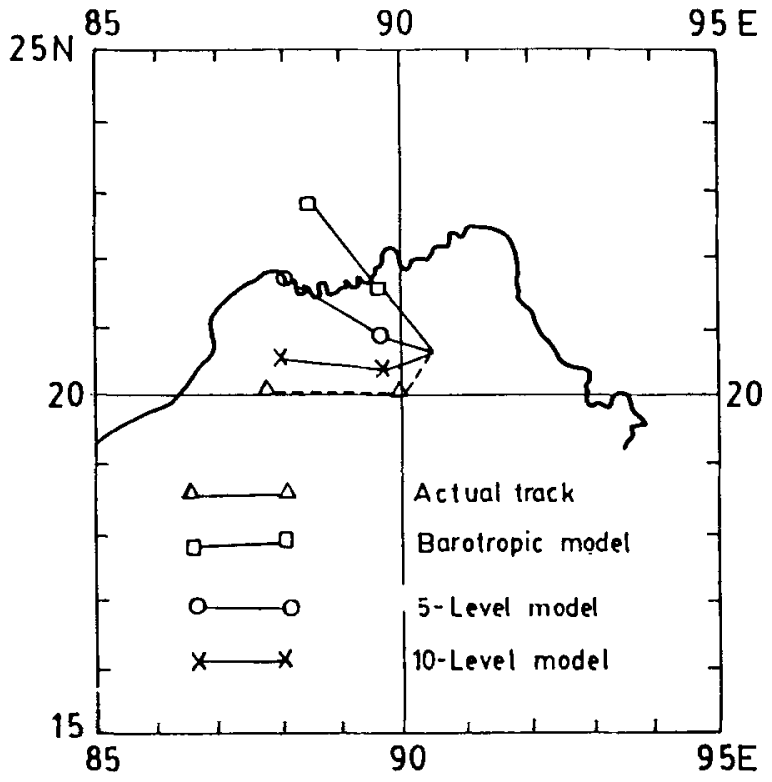

Figure 3. Performance of the barotropic and multi-level (5-layer and 10-layer) primitive equation regional models (LAM) to predict the track of a monsoon depression over the Bay of Bengal.

experiment (MONEX)-79 from July 1979, and the corresponding forecast tracks of the disturbance obtained from the barotropic PE model (described in $\S 3.1 \mathrm{~b}$ ) and the multi-level PE model with 5 and 10 vertical levels, are shown in figure 3 . In all these cases, the horizontal domain of the model is bounded by $30^{\circ} \mathrm{E}-105^{\circ} \mathrm{E}$ and $7.5^{\circ} \mathrm{S}-45^{\circ} \mathrm{N}$ with a grid resolution of $1.875^{\circ}$. For this study the FGGE level-IIIb analysis of the European Centre for Medium Range Weather Forecasts, UK obtained as a result of the First GARP Global Experiment (FGGE) and MONEX-79, which is considered to be the first-ever excellent database for numerical studies on the summer monsoon, was obtained to constitute the initial conditions. Track prediction based on the integral method with barotropic, 5-level and 10-level PE models clearly demonstrates the impact of a better representation of realistic atmosphere and surface processes including topography on the improvement of such forecasts.

(ii) General circulation model (GCM) - General circulation models over the entire globe do not require lateral boundary conditions. Such models are generally used for extended range simulation/prediction of the atmospheric processes. In this study, the operational global model of the European Centre for Medium Range Weather Forecasts (ECMWF), UK was used to illustrate its performance in the prediction of storm tracks in the Bay of Bengal. A detailed description of the T-63 spectral GCM is given by Simmons \& Jarraud (1984) and performance of the model with modified parameterization of the physical processes in predicting the 1979 summer monsoon onset is presented by Slingo et al (1988). The model is used to predict the track of two monsoon depressions in the Bay of Bengal during 23 June-26 June, 1979 and 28 June-1 July, 1979. The observed and predicted tracks are shown in figure 4. For this study, the FGGE level-IIIb data set was used as initial conditions. While the forecasts for the tracks of monsoon disturbances upto 72 hours are reasonably close to the observed tracks, a large difference in the determination of initial positions of the disturbances is mainly attributed to lack of adequate and accurate observations in their vicinity in data-sparse oceanic regions. 


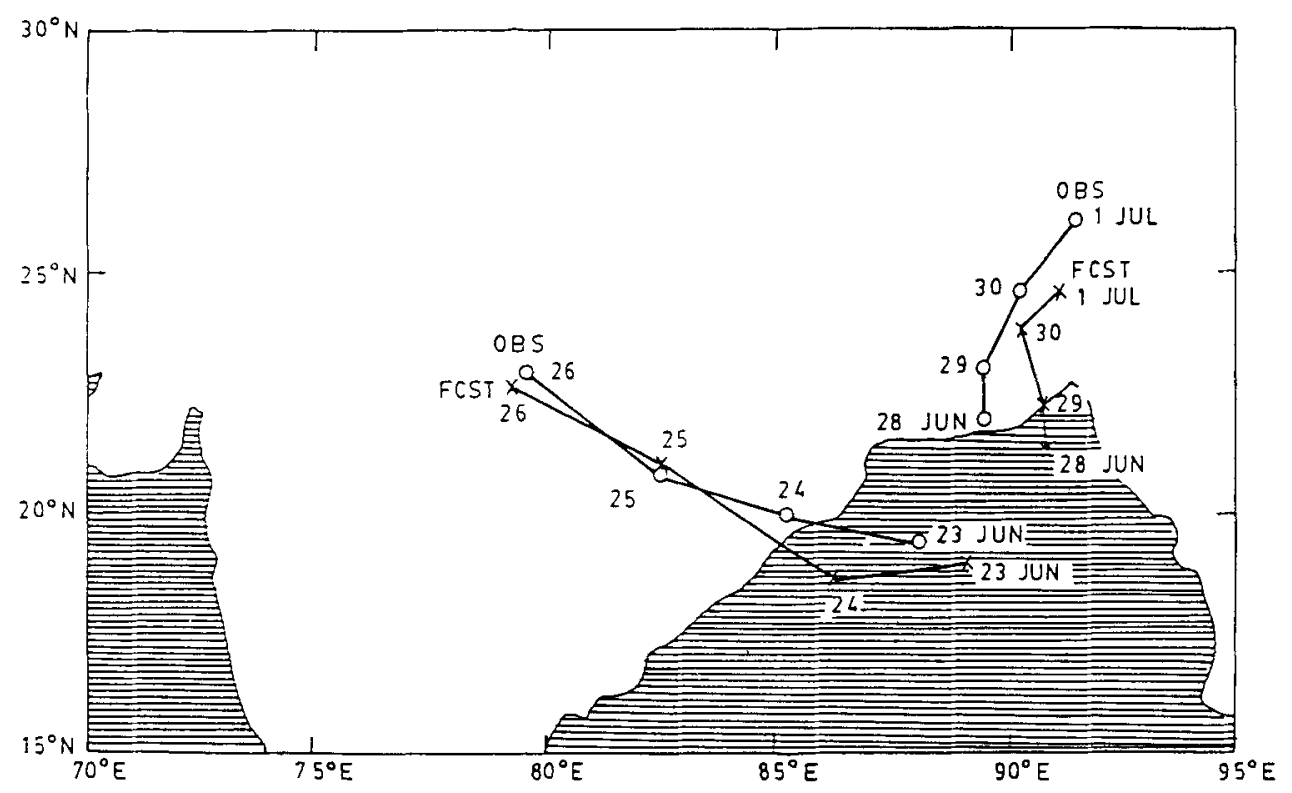

Figure 4. Performance of a multi-level global spectral modei (GCM) to predict the track of a monsoon depression over the Bay of Bengal.

3.1d Limitations and prospects of numerical methods: Considerable success has been achieved in predicting large scale atmospheric motions using numerical weather prediction models, largely owing to the availability of high-speed supercomputers, space-based observation systems, data assimilation, initialization, sophisticated numerical techniques and better understanding of various physical processes and their parameterization in Numerical Weather Prediction (NWP) models. However, improvement in prediction of tropical cyclones using NWP models has been sluggish mainly due to lack of accurate and adequate data coverage over tropical oceans, which are the breeding grounds of tropical cyclones. Furthermore, we do not as yet have a clear understanding of their thermodynamics, particularly, parameterization of convection, cloud-radiation, boundary layer, air-sea interaction and their feedback processes. Future improvements in NWP for prediction of the tracks of tropical cyclones/disturbances actually call for the following developments.

(i) Augmented coverage of observing systems over tropical oceans.

(ii) Optimum utilization of non-conventional data sets on tropical cyclones obtained from satellites, weather radar and aircraft etc. in the preparation of initial data for NWP models.

(iii) Production of synoptic data sets on wind, temperature and moisture in the vicinity of tropical cyclones from their observed characteristic features (synthetic data generation).

(iv) Appropriate diabatic and physical initialization procedures to retain/strengthen the realistic intensity of tropical cyclones (better representation of divergent flow and moisture fields).

(v) Nudging the centre of the observed cyclones with that of the objectively analysed centres to be served as initial conditions for NWP models. 
(vi) Use of realistic sea surface temperature and topography data as lower boundary conditions in the NWP models.

(vii) Development of high resolution regional/global models with horizontal resolutions of about $30-40 \mathrm{~km}$ and discretized for $25-30$ vertical levels, for better representation of tropical cylones in the NWP models.

(viii) Improvement in the parameterization of the physical processes in the NWP model, in particular, convection, cloud radiation, boundary layer and air-sea exchange processes and their feedback mechanisms in association with tropical cyclones.

\subsection{Statistical methods}

Tropical storms move under the influence of both external and internal forces. External forces refer to the effect of large-scale atmospheric processes on their movement which is mainly manifested through steering flow. Internal forces arise from the storm itself and from its interaction with the steering flow in the troposphere.

Different techniques and predictors are used to consider these forces directly or indirectly in formulating statistical methods for forecasting the movement of tropical storms. In general, linear and nonlinear regression analyses are widely used. Potential predictors may be classified into four categories: synoptic, inertial, climatological and those obtained from large-scale meteorological fields through empirical functions (like empirical orthogonal function). Statistical methods differ from one another mainly by the statistical techniques and predictors used in their formulation.

In the present work, we propose four different statistical methods to predict the movement of post-monsoon season storm tracks in the Bay of Bengal. A brief description of these four methods is given below.

Method 1 (Mohanty 1979): This method is formulated on the basis of synoptic predictors. The sea level pressure field $\left(P_{0}\right)$, geopotential fields at 700 and $500 \mathrm{hPa}$ pressure levels $\left(H_{700}\right.$ and $\left.H_{500}\right)$ and the 1000 to $700 \mathrm{hPa}$ and 700 to $500 \mathrm{hPa}$ thickness are the current synoptic fields which are obtained over a regular $5^{\circ}$ latitude/longitude moving coordinate, $7 \times 8$ grid system, at the centre of a cyclonic storm. Other parameters taken into consideration are geostrophic steering currents at 700 and $500 \mathrm{hPa}$ surfaces and the preceding 12 hour track of the storm centre $\left(S_{x}, S_{y}\right)$. A stepwise screening procedure is used to obtain a series of regression equations for advance computations of the zonal and meridional motions for periods of $0-12,12-24,24-36$ and $36-48 \mathrm{~h}$.

Method 2 (Kivganov \& Mohanty 1978): This method makes use of the first five coefficients of empirical orthogonal functions (e.o.f.) of the meteorological fields $P_{0}$, $H_{700}, H_{500}$ and 100 to 700 and 700 to $500 \mathrm{hPa}$ thickness as potential predictors, instead of the actual meteorological fields. Additional prediction parameters used are the empirical orthogonal functions of the e.o.f. coefficients of $P_{0}, H_{700}$ and $H_{500}$. Such a second expansion of e.o.f. allows one to incorporate the interaction in vertical co-ordinates. Further, the persistence of the systems $S_{x}$ and $S_{y}$ are also used as predictors. A set of prediction equations similar to those of method 1 are obtained by stepwise regression analysis.

Method 3 (Kivganov \& Mohanty 1979a): This method is based on empirical predictors only (i.e. climatology and persistence) and is similar to those developed for other 
regions (Neumann 1972, Hope \& Neumann 1973, Neumann \& Randrianarison 1976. and others). Seven basic predictors used in this scheme are: day number, latitude and longitude of the centre of the storm, corresponding to current time $t_{0}$, and average zonal and meridional speed of the cyclone over the previous 12 and 24-hour period. Third-order polynomial equations, consisting of seven primary and 112 secondary predictors are subjected to stepwise screening procedures to obtain a set of prediction equations as in methods 1 and 2.

Method 4 (Mohanty 1980): This method is based on the principles discussed in the work of Neumann et al (1972). First, three independent sets of forecasts, derived from the above three methods (methods 1-3) are obtained. These are then used as basic predictors to derive a new set of prediction equations. In order to consider the nonlinear effects, a third-order polynomial with 3 basic predictors and 16 additional predictors are generated. A stepwise screening regression procedure is used to eliminate the predictors which fail to provide the prescribed minimum incremental reductions of variance $\left(0 \cdot 1^{\circ}{ }_{0}\right)$. Prediction equations are derived from different sources as used in methods 1,2 and 3.

\section{Data}

For this work, using dynamical models we used vertically averaged wind fields at $850,700,500,300$ and $200 \mathrm{hPa}$ pressure levels. The geopotential field was derived from wind fields by calculating stream functions and subsequently by reverse balance equations. Such a procedure for obtaining the geopotential field is found to be quite satisfactory (Mohanty \& Madan 1983). Data were obtained over a rectangular region $\left(3^{-}-40^{-} \mathrm{N}\right.$ and $\left.60^{-}-120^{\circ} \mathrm{E}\right)$ with a regular grid of length $210 \mathrm{~km}$ for 14 cases of post-monsoon storms in the years 1975 and 1976. Five-year data samples of cyclonic storms from 1970 to 1974 were used to obtain prediction equations using statistical methods 1 and 2. while 98-year climatological data (1877-1974) were used for method 3 . For method 4, the predicted values of zonal and meridional motions obtained by statistical methods 1, 2 and 3, above, were used on a homogeneous, reliable data set, consisting of 40 cases of post-monsoon storms (1970 to 1974). Performance tests of these four statistical methods were carried out on an independent data sample, comprising 14 cases of post-monsoon TS and STS in 1975 and 1976. In this study, a case of the storm is referred to 2-day trajectories. Thus, if a cyclonic storm continues for a period of 3 days, it provides 2 cases for study of 48 -hour forecasts (day 1-2 and day $2-3$ ). The data requirement and data set used for multi-level NWP models are discussed in $\$ 3 \cdot 1 \mathrm{c}$.

\section{Results and discussion}

Performance of the objective methods is measured in terms of the distance between the forecast and observed positions of the centre of the storm. referred to as the error vector $(\Delta \mathbf{R})$. The mean vector error is estimated by:

$$
\Delta \mathbf{R}=\frac{1}{N} \sum_{i=1}^{N}\left(\Delta C X_{i}^{2}+\Delta C Y_{i}^{2}\right)^{1 / 2}
$$


where $N$ is the number of cases and $\Delta C X$ and $\Delta C Y$ are zonal and meridional errors respectively.

Here, our study will be confined to only two dynamical models: the steering flow model $(\$ 3 \cdot 1 \mathrm{a})$ and the barotropic integral model $(\$ 3 \cdot 1 \mathrm{a})$ to compare their performances with those of the four statistical methods $(\$ 3 \cdot 2)$. As adequate data sets were not available for more complex dynamical modelling (\$3.1) for 1975-76, these models will not be considered here. The performance of the complex LAM and GCM have already been described in $\$ 3 \cdot 1 \mathrm{c}$ in respect of tropical disturbances with FGGE level III-b data sets of 1979.

In order to investigate the performance of the six objective methods discussed in $\S 3$, mean vector errors were obtained from 14 cases of the post-monsoon storms in the Bay of Bengal over a two-year period (1975-1976) for different forecast time intervals as illustrated in figure 5. Clearly, the performance of the statistical method 4 is better as compared with those of others over all the time intervals (0-12 to 36-48 hours) of forecast. Performance of the integral method is quite satisfactory and better than those of the remaining four methods examined in this study. It is found that in relative terms, performance of the statistical methods in shorter time intervals of up to 24 hours is superior to those of numerical methods, but for longer time intervals the dynamical methods prove better.

Performances of the six objective methods, proposed in this work, for forecasting the movement of the post-monsoon cyclones in the Bay of Bengal were compared with some of the existing models for this region (Sikka \&.Suryanarayan 1972; Bansal \& Dutta 1974; Sikka 1975) and found to be quite satisfactory. However, such a comparison is not truly representative as these other methods treated both pre- and post-monsoon storms and used different sample lengths. Prediction of the track with a point of

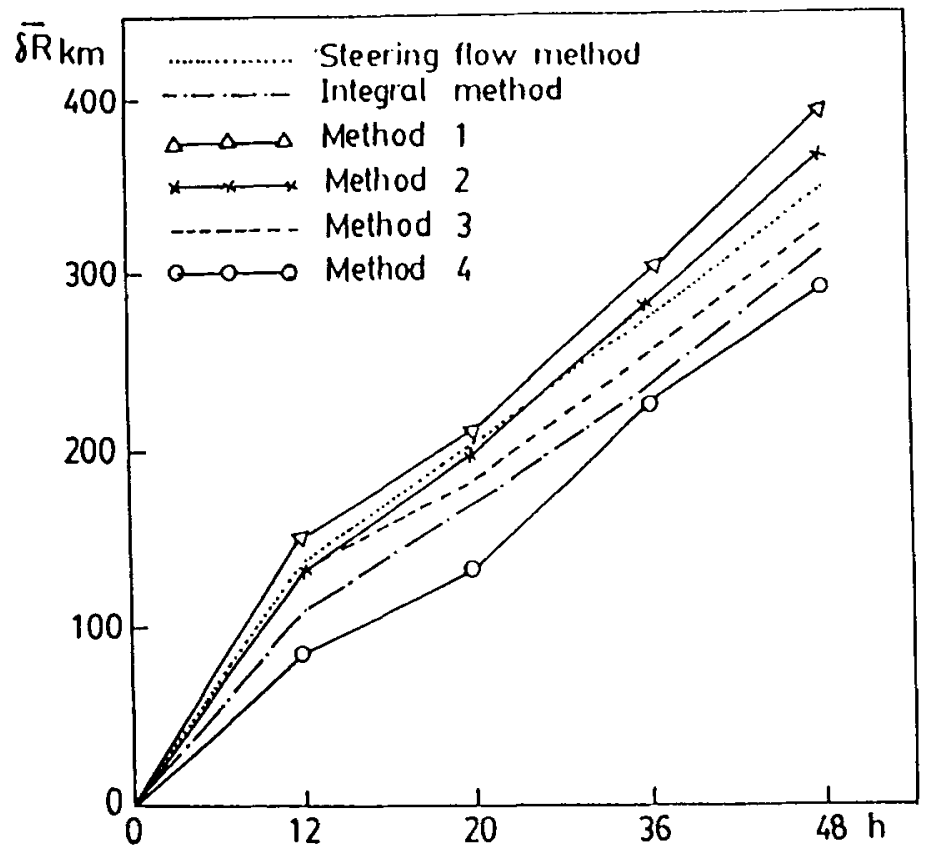

Figure 5. Performance (average magnitude of vector error) of specified objective methods in prediction of tracks of post-monsoon cyclones over the Bay of Bengal. 
recurvature, which occurs mainly in the post-monsoon season, is quite a difficult task; but considering all these aspects, statistical method 4 is found to be appreciably better than others.

\section{Conclusions}

Based on the results presented in this study, the following general conclusions are drawn.

(i) Although, two severe cyclonic storm seasons are generally observed in the Bay of Bengal, the primary one is the post-monsoon period. Also, it is during this season that the maximum number of complex tracks (i.e. recurved path of cyclonic storm motion) develop whose prediction is quite difficult.

(ii) The dynamical approach based on the integral method is found to be the most attractive for predicting the motion of tropical cyclones, the complex multi-level PE models being the most suitable ones.

(iii) In the present context, however, with lack of adequate meteorological data in and around the tropical cyclones and inaccuracies in parameterization of their physical processes, the integrated statistical approach (method 4 ) is found to be more satisfactory than the dynamical methods and other statistical methods discussed in this paper.

However, the predictive potential of dynamical models which are now greatly improved can be exploited with great effect with improved data coverage, generation of synthetic data, diabatic initialization and more accurate parameterization of physical processes.

\section{References}

Bansal R K, Datta R K 1974 A statistical method of forecasting the movement of cyclonic storms in the Bay of Bengal. Indian J. Meteorol. Geophys. 25: 391-397

De Angelis D 1976 World of tropical cyclones - North Indian Ocean. Mar. Weather Log. 20: 191-194

Gray W M 1968 Global view of the origin of tropical disturbances and storms. Mon. Weather Rev. 96: 669-700

Hope J R, Neumann C J 1973 Operational use of the NHC-72 tropical cyclone forecast system: In Program of the 8th Technical Conference on Hurricanes and Tropical Meteorology. Bull. Am. Meteorol. Soc. 54: 147-188

Hope J R, Neumann C J 1977 A survey of world-wide tropical cyclones prediction models. Proceeding 11th American Meteorological Society Tech. Conference on Hurricane and Tropical Meteorology (Am. Meteorol. Soc.) pp. 367-372

India Met. Dept. 1979 Tracks of storms and depressions in the Bay of Bengal and the Arabian Sea, 1877-1970, India Meteorological Department

Kasahara A 1959 A comparison between geostrophic and non-geostrophic numerical forecasts of hurricane movement with the barotropic steering model. J. Meteorol. 16: 377-384

Kivganov A F, Mohanty U C 1978 On the physical statistical method for forecasting the movement of tropical storms in the Bay of Bengal. Meteorol. Hydrol. 5: 19-25

Kivganov A F, Mohanty U C 1979a On the application of nonlinear regression analysis to forecast the movement of tropical cyclones in the Bay of Bengal. Tr. Hydrometeorol. Centre, USSR 125: 47-58 
Kivganov A F, Mohanty U C 1979b Hydro-dynamical method of forecasting the trajectories of tropical cyclones in the Indian seas. Meteorol., Climatol. Hydrol. 15: 9-15

Minina L S, Niguen V N 1969 Tropical cyclones of Indian Ocean. Tr. Hydrometeorol. Centre, USSR 41: $29-50$

Mohanty U C 1978 Tropical cyclones in the Bay of Bengal and objective methods for prediction of their movement. Ph D thesis, Odessa Hydro-Meteorological Institute, USSR

Mohanty U C 1979 Statistical method of forecasting tropical cyclones. Meteorol. Climatol. Hydrol. 15: 16-22

Mohanty U C 1980 An objective method of forecasting the movement of tropical cyclones in the Bay of Bengal based on a number of statistical models. WMO Symposium on Probabilities and Statistical Methods in Weather Forecasting, Nice, France (Geneva: WMO) pp. 359-364

Mohanty U C 1982 Some characteristics of dynamical initialization of mass and velocity fields in the lower latitudes. Mausam 33: 29-34

Mohanty U C, Dube S K 1981 Statistical structure of the meteorological parameters over the Bay of Bengal during Monsoon-77 experiments. Mausam 32: 315-320

Mohanty U C, Madan S C 1983 A numerical method for computing stream function and velocity potential. Mausam 34: $375-382$

Mohanty U C, Paliwal R K, Tyagi A, John A 1989 Evaluation of a multi-level primitive equations limited area model for short range prediction over Indian region. Mausam 40: $34-42$

Mohanty U C, Paliwal R K, Tyagi A, Sarin V B 1988 A suitable scheme of dynamic initialization for a multi-level primitive equations model in tropics. Mausam 39: 139-148

Neumann C J 1972 An alternate to the Hurran tropical cyclone forecast system - NOAA Tech. Memo. NWS SR-62

Neumann C J, Hope J R, Miller B I 1972 Statistical method for combining synoptic and empirical prediction systems. NOAA Tech. Memo. NWS SR-63

Neumann C J, Randrianarison E W 1976 Statistical prediction of tropical cyclone motion over the South-West Indian Ocean. Mon. Weather Rev. 104: 76-85

Sanders F, Pike A C, Gaertner J P 1975 A barotropic model for operational prediction of tracks of tropical storms. J. Appl. Meteorol. 14: 256-280

Sikka D R 1975 Forecasting the movement of tropical cyclones in the Indian seas by non-divergent barotropic model. Indian J. Meteorol. Geophys. 26: 323-325

Sikka D R, Suryanarayan R 1972 Forecasting the movement of tropical storms/depressions in the Indian region by a computer oriented technique using climatology and persistence. Indian J. Meteorol. Geophys. 23: 35-40

Simmons A J, Jarraud M 1984 The design and performance of the new ECMWF operational model. ECMWF Seminar on Numerical Methods for Weather Prediction (Reading: ECMWF) 2: $1-59$

Singh S S, Saha K 1976 Numerical experiments with a primitive equation barotropic model for prediction of movement of monsoon depressions and tropical cyclones. J. Appl. Meteorol. 15: $805-810$

Sitnikov E G 1968 Numerical experiments on prediction of track of tropical cyclones in the Republic of Cuba. Tr. Hydrometeorol. Centre, USSR 29: 21-32

Slingo J M, Mohanty U C, Tiedtke M, Pearce R P 1988 Prediction of the 1979 summer monsoon onset with modified parameterization schemes. Mon. Weather Rev. 116: 328-346 